\title{
Real-time surveillance for chronic conditions in Massachusetts using EHR data
}

\author{
Noelle Cocoros ${ }^{\star 1}$, John Menchaca ${ }^{1}$ and Michael Klompas ${ }^{1,2}$ \\ 'Population Medicine, Harvard Medical School, Harvard Pilgrim Health Care Institute, Boston, MA, USA; 'Brigham and Women's \\ Hospital, Boston, MA, USA
}

\section{Objective}

To assess the feasibility of tracking the prevalence of chronic conditions at the state and community level over time using MDPHnet, a distributed network for querying electronic health record systems

\section{Introduction}

Public health agencies and researchers have traditionally relied on the Behavioral Risk Factor Surveillance System (BRFSS) and similar tools for surveillance of non-reportable conditions. These tools are valuable but the data are delayed by more than a year, limited in scope, and based only on participant self-report. These characteristics limit the utility of traditional surveillance systems for program monitoring and impact assessments. Automated surveillance using electronic health record (EHR) data has the potential to increase the efficiency, breadth, accuracy, and timeliness of surveillance. We sought to assess the feasibility and utility of public health surveillance for chronic diseases using EHR data using MDPHnet. MDPHnet is a distributed data network that allows the Massachusetts Department of Public Health to query participating practices' EHR data for the purposes of public health surveillance (www.esphealth.org). Practices retain the ability to approve queries on a case-by-case basis and the network is updated daily.

\section{Methods}

We queried the quarterly prevalence of pediatric asthma, smoking, type 2 diabetes, obesity, overweight, and hypertension statewide and in 9 Massachusetts communities between January 1, 2012 and July 1,2016 . We selected these 9 communities because they were participating in a state-funded initiative to decrease the prevalence of one or more of these conditions. Conditions were defined using algorithms based upon vital signs, diagnosis codes, laboratory measures, prescriptions, and self-reported smoking status. Eligible patients were those with at least 1 encounter of any kind within the 2 years preceding the start of each quarter. Results were adjusted for age, sex, and race / ethnicity using the 2010 Massachusetts census data.

\section{Results}

Surveillance data were available for 1.2 million people overall, approximately $20 \%$ of the state population. Coverage varied by community with $>28 \%$ coverage for 7 of the communities and $11 \%$ coverage in the eighth. The ninth community had only $2 \%$ coverage and was dropped from further analyses. The race / ethnicity distribution in MDPHnet data was comparable to census data statewide and in most communities. Queries for all six conditions successfully executed across the network for all time periods of interest. The prevalence of asthma among children under 10 yrs rose from $12 \%$ in January 2012 to $13 \%$ in July 2016 . Current smoking in adults age $\geq 20$ rose from $14 \%$ in 2013 to $16 \%$ in 2016 (we excluded results from 2012 due to changes in documentation propelled by the introduction of meaningful use criteria). This is comparable to the $15 \%$ rate of smoking per BRFSS in $2014^{1}$. Obesity among adults increased slightly from $22 \%$ to $24 \%$ during the study period, results nearly identical to the most recent BRFSS results for Massachusetts
( $23 \%$ in 2014 and $24 \%$ in 2015$)^{2}$. The prevalence of each condition varied widely across the communities under study. For example, for the third quarter of 2016, the prevalence of asthma among children under 10 ranged from $5 \%$ to $23 \%$ depending on the community, the prevalence of smoking among adults ranged from $11 \%$ to $35 \%$, and the prevalence of type 2 diabetes among adults ranged from $7 \%$ to $14 \%$. We also examined differences in disease estimates by race / ethnicity. Substantial racial / ethnic differences were evident for type 2 diabetes among adults, with whites having the lowest prevalence at $7 \%$ and blacks having the highest at $12 \%$ in the third quarter of 2016; this trend was consistent over the study period.

\section{Conclusions}

Our study demonstrates that MDPHnet can provide the Massachusetts Department of Public Health with timely populationlevel estimates of chronic diseases for numerous conditions at both the state and community level. MDPHnet surveillance provides prevalence estimates that align well with BRFSS and other traditiona surveillance sources but is able to make surveillance more timely and more efficient with more geographical specificity compared to traditional surveillance systems. Our ability to generate real-time time-series data supports the use of MDPHnet as a source for project/ program evaluation.

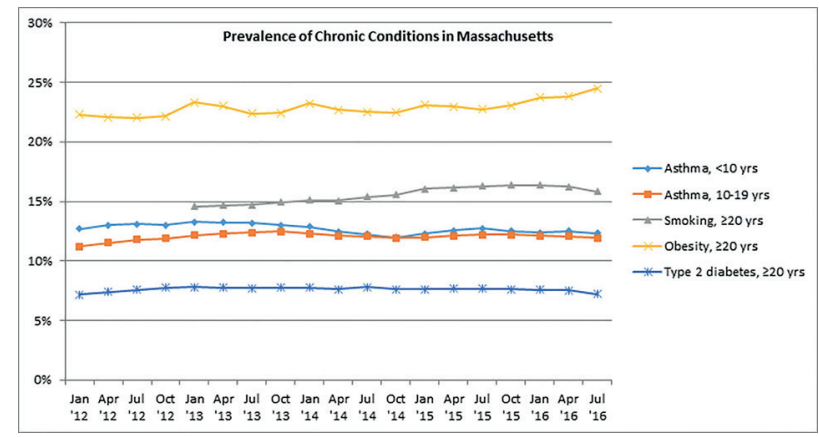

\section{Keywords}

Asthma; Smoking; Obesity; chronic disease; real-time surveillance

\section{Acknowledgments}

Thank you to our colleagues at Commonwealth Informatics.

\section{References}

1. Massachusetts Dept. of Public Health, 2015. A Profile of Health Among Massachusetts Adults, 2014, Results from the Behavioral Risk Factor Surveillance System, http://www.mass.gov/eohhs/docs/ dph/behavioral-risk/report-2014.pdf, accessed 9/6/2016.

2. Robert Wood Johnson Foundation, 2016. The State of Obesity 2016 http://stateofobesity.org/states/ma/, accessed 9/6/2016.

\section{*Noelle Cocoros}

E-mail: noelle_cocoros@harvardpilgrim.org 